\title{
Management of conjunctivitis during the COVID-19 pandemic 2020
}

Jane Khan, Heather G Mack

AUSTRALIA is experiencing an epidemic of COVID-19 due to the SARS-CoV-2 virus. Conjunctivitis may be a feature of this illness, with tears containing SARS-CoV-2 virus, and a source of potential transmission via aerosols. General practitioners (GPs) frequently see patients with red eyes and need to be aware of the necessary precautions to reduce spread to themselves and the community.

In December 2019, SARS-CoV-2 first emerged in Wuhan, China. Symptoms are often mild (80\%), with fever, cough, occasional shortness of breath and, in rare cases, diarrhoea. Up to $5 \%$ of patients develop critical disease with respiratory failure or multiorgan dysfunction. There is no vaccine or specific antiviral treatment available; treatment is limited to supportive care.

Little is known about the ophthalmic features of coronaviruses in general. Animal models show a wide spectrum of disease from conjunctivitis and anterior uveitis, to chorioretinal inflammation and degeneration and optic neuritis. ${ }^{1}$ There are sparse reports of patients infected with other coronaviruses (eg HCoV-NL63) developing acute bilateral follicular conjunctivitis; RNA from SARS-CoV-1 was detected in tears, ${ }^{2}$ although conjunctivitis was not a common feature of severe acute respiratory syndrome (SARS).

SARS-CoV-2 is droplet spread and viable following aerosolisation for up to three hours. ${ }^{3}$ SARS-CoV-2 has been detected at a low rate in tears. ${ }^{4}$ However, in this study of patients with positive reverse transcription polymerase chain reaction (RT-PCR) sputum samples, only one patient with conjunctivitis yielded positive RT-PCR results from a conjunctival swab.
However, 58 conjunctival swab samples from 29 patients with positive sputum samples were all negative. A further study of 17 cases (one with some ocular redness) showed no PCR or cytopathological effect of SARS-CoV-2 in tear samples even when the nasopharyngeal swabs were positive. ${ }^{5}$ There have been reports that procedures causing aerosolisation of ocular adnexa are high risk for transmission, but these are surgical procedures performed by otolaryngologists or oculoplastic surgeons using endonasal techniques. Taken together, these data raise the possibility of spread of SARS-CoV-2 via tears and highlight the risk of eye examinations as an aerosol-generating procedure with potential risk to healthcare workers.

It is thought that patients with SARS-CoV-2 may develop eye redness and watering, or conjunctivitis indistinguishable from typical viral conjunctivitis, rarely as the presenting complaint. Conjunctivitis is a common presenting problem for GPs and, despite the likely low risk of viral shedding from the conjunctiva, it is important to reduce risk while caring for these patients. If possible, patients should be triaged prior to attending the clinic. Those with conjunctivitis who are at high risk of COVID-19 infection should be directed to tertiary hospitals with COVID-19 facilities and ophthalmology departments. Patients considered to be low risk should be managed by telemedicine consultation. If conjunctivitis is unilateral and sticky, it would be reasonable to consider topical antibiotics (eg chloramphenicol drops four times daily for four days). If conjunctivitis is bilateral and watery, it is more likely to be viral, and symptomatic treatment with regular hygiene and artificial tears is recommended. If symptoms persist, it is appropriate to consider a mild steroid (eg fluorometholone drops four times daily for four days). If at any time the patient develops a reduction in vision, significant pain or lack of improvement with topical antibiotics and/or steroids, they should be referred for ophthalmic care, preferably by telemedicine consultation.

Patients considered to be at high risk of COVID-19 who arrive unannounced with conjunctivitis should have a minimum eye examination performed, with healthcare workers wearing full personal protective equipment (PPE) including protective eyewear, and the room disinfected after departure. Slit lamp shields are recommended as additional protective equipment. If follow-up is necessary, this should be done by telemedicine; patients with significant visual impairment should be referred directly to definitive ophthalmology care to conserve PPE and reduce patient travel in the community. Many ophthalmologists are now offering telemedicine consultations.

Data are limited, and the risk may be low. However, prudence suggests that, during the pandemic, GPs consider all patients with conjunctivitis in the setting of an upper respiratory tract infection or typical viral conjunctivitis to have SARS-CoV-2 infection until proven otherwise, and take appropriate precautions wherever possible.

First published online 1 May 2020.

\footnotetext{
Authors

Jane Khan MBBS, FRCOphth, FRANZCO, MD, Chair of Public Health Committee, The Royal Australian and New Zealand College of Ophthalmologists, NSW; Clinical Senior Lecturer, University of Western Australia, WA; Consultant Ophthalmologist, Royal Perth Hospital, WA

Heather G Mack PhD, FRANZCO, Clinical Associate Professor, Department of Surgery (Ophthalmology), University of Melbourne, Vic.

hmack@eyesurgery.com.au

Competing interests: None.
} 
Provenance and peer review: Commissioned, peer reviewed.

\section{Acknowledgements}

The authors acknowledge the contributions of the Royal Australian and New Zealand College of Ophthalmologists Public Health committee, Fellows and staff.

Citation: Khan J, Mack HG. Management of conjunctivitis during the COVID-19 pandemic 2020. Aust J Gen Pract 2020;49 Suppl 18. doi: 10.31128/ AJGP-COVID-18.

\section{References}

1. Seah I, Agrawal R. Can the coronavirus disease 2019 (COVID-19) affect the eyes? A review of coronaviruses and ocular implications in humans and animals. Ocul Immunol Inflamm 2020;28(3):391-95. doi: 10.1080/09273948.2020.1738501.

2. Loon S-C, Teoh SCB, Oon LLE, et al. The severe acute respiratory syndrome coronavirus in tears. Br J Ophthalmol 2004;88(7):861-63. doi: 10.1136/ bjo.2003.035931.

3. van Doremalen N, Bushmaker T, Morris DH, et al. Aerosol and surface stability of SARS CoV-2 as compared with SARS-CoV-1. N Engl J Med 2020;382(16):1564-67. doi: 10.1056/ NEJMc2004973.

4. Xia J, Tong J, Liu M, Shen Y, Guo D. Evaluation of coronavirus in tears and conjunctival secretions of patients with SARS-CoV-2 infection. J Med Virol 2020. doi: 10/1002/jmv.25725.

5. Seah IYJ, Anderson DE, Kang AEZ, et al. Assessing viral shedding and infectivity of tears in coronavirus disease 2019 (COVID-19) patients. Ophthalmology 2020;S0161-6420(20)30311-0. doi: 10.1016/j.ophtha.2020.03.026. 